\title{
Implante Percutâneo de Prótese Valvular Aórtica: Progressos e Desafios de uma Nova Era da Cardiologia Intervencionista
}

\author{
Ver artigos relacionados \\ nas páginas 24 e 28
}

Dimytri A. A. Siqueira',

Alexandre A. C. Abizaid' ${ }^{1}$, J. Eduardo Sousa ${ }^{1}$
$\mathbf{R}$ ealizado pela primeira vez em 2002, o implante percutâneo de bioprótese aórtica tem despertado significativo interesse nos últimos anos, por tratar-se de alternativa segura e menos invasiva para pacientes portadores de estenose aórtica e alto risco cirúrgico. Atualmente, dois tipos de prótese são disponíveis e comercializadas na Europa e diversas outras estão sendo investigadas, e mais de 20 mil pacientes foram tratados até 2010. Vários registros têm sido publicados e importantes estudos randomizados e comparativos com a cirurgia de troca valvar e com o tratamento conservador estão disponíveis (SOURCE, PARTNER) ou em andamento (UK TAVI Trial, SURTAVI). Em nosso País, cerca de 200 pacientes já foram submetidos ao implante da bioprótese autoexpansível CoreValve ${ }^{\mathrm{TM}}$ (Medtronic Inc., Minneapolis, Estados Unidos), e vislumbra-se, ainda no primeiro semestre, o início da utilização da bioprótese balão-expansível Edwards-SAPIEN ${ }^{\top M}$ (Edwards Lifesciences, Irvine, Estados Unidos).

Nesta edição da Revista Brasileira de Cardiologia Invasiva, somos apresentados a duas das experiências nacionais de implante valvular aórtico percutâneo. Lemos et al. ${ }^{1}$ descrevem os resultados clínicos dos primeiros 75 pacientes tratados com a prótese CoreValve $^{\mathrm{TM}}$ em dois centros, no Brasil $(n=14)$ e na Itália ( $\mathrm{n}=61$ ); e Bernardi et al. ${ }^{2}$, por sua vez, relatam a evolução de dois anos dos primeiros 4 pacientes submetidos ao procedimento no Sul do País. Os resultados das duas publicações exprimem tanto os notáveis progressos alcançados como os grandes desafios a serem transpostos para a ampliação nas indicações desse procedimento.

\section{Progressos obtidos}

Em menos de 10 anos, testemunhamos importantes avanços como a redução do perfil dos dispositivos, a utilização de vias de acesso alternativas à via femoral (transubclávia, transaórtica, apical), o aperfeiçoamento da técnica de implante e a busca pela normatização dos desfechos a serem utilizados nos estudos clínicos futuros (conforme propósito do Valve Academic Research Consortium - $V A R C^{3}$ ). Contudo, o que nos impressiona são os resultados clínicos obtidos.

As evidências atuais demonstram que as técnicas para o tratamento percutâneo da estenose aórtica são efetivas e promovem benefícios clínicos e hemodinâmicos em pacientes de alto risco ou com contraindicação cirúrgica. Ao se analisar os gradientes e a área valvar alcançados em diversos estudos, depreende-se que o desempenho das próteses percutâneas atualmente utilizadas é comparável ao das próteses cirúrgicas. Em recente investigação, Clavel et al. ${ }^{4}$ sugerem inclusive que os parâmetros hemodinâmicos após o implante da prótese Edwards-SAPIEN ${ }^{\mathrm{TM}}$ podem ser até mais favoráveis que os observados com as próteses cirúrgicas, o que resulta em melhoria mais pronunciada da fração de ejeção em pacientes com disfunção ventricular esquerda. O acompanhamento tanto clínico como ecocardiográfico a médio prazo apresenta ainda a preservação dos resultados obtidos, como bem demonstrado no artigo de Bernardi et al. ${ }^{2}$

Se no início da experiência mundial - com a utilização de acesso anterógrado - as taxas de sucesso do procedimento eram de $80 \%$, nas séries mais recentes os índices são superiores a 95\%., . $^{-1} \mathrm{Na}$ experiência conjunta Brasil-Itália, a taxa de sobrevida global aos 30 dias foi de $91,6 \%$, concordante com os achados de registros internacionais e multicêntricos ${ }^{7-9}$, que incluíram pacientes submetidos ao implante das próteses Edwards-SAPIEN ${ }^{\mathrm{TM}}$ e CoreValve ${ }^{\mathrm{TM}}$ e que revelam taxas de sobrevida aos 30 dias de $89 \%$ a 95\%. A mortali-

\footnotetext{
1 Instituto Dante Pazzanese de Cardiologia - São Paulo, SP, Brasil.

Correspondência: Alexandre A. C. Abizaid. Av. Dr. Dante Pazzanese, 500 - Vila Mariana - São Paulo, SP, Brasil - CEP 04012-909 E-mail: aabizaid@uol.com.br

Recebido em: 20/3/2011 • Aceito em: 21/3/2011
} 
dade observada nesses estudos é sistematicamente menor que a mortalidade cirúrgica estimada por escores (EurOSCORE e STS-PROM).

Notáveis ainda são os resultados do estudo randomizado PARTNER $\mathrm{B}^{10}$, que estabeleceram o tratamento percutâneo da valva aórtica à condição de única forma de terapia a demonstrar consistente redução de mortalidade em pacientes com estenose aórtica grave sintomática e risco cirúrgico proibitivo. Nesse estudo, 358 pacientes com contraindicação cirúrgica foram randomizados para estratégia conservadora ou para implante da prótese Edwards-SAPIEN ${ }^{\mathrm{TM}}$ : nos pacientes submetidos a tratamento percutâneo, a mortalidade aos 30 dias foi de 6,4\%. Após um ano, foi observada significativa redução da mortalidade nesses pacientes, quando comparada aos indivíduos mantidos em tratamento clínico $(30,7 \%$ vs. 50,7\%; P < 0,001). Tal magnitude de benefício clínico encontra poucos precedentes na história da cardiologia.

\section{Progressos almejados}

Embora os resultados clínicos em pacientes de alto risco sejam bastante favoráveis, diversos aspectos ainda necessitam de entendimento e vários problemas devem ser solucionados antes que as indicações sejam estendidas a pacientes de menor risco. Dentre os desafios, destacam-se a insuficiência aórtica paraprotética, o acidente vascular cerebral (AVC) isquêmico e a necessidade de marca-passo definitivo, todos observados nos artigos em questão.

O implante percutâneo de prótese aórtica associa-se mais frequentemente à ocorrência de regurgitação paraprotética que a cirurgia de troca valvar. O refluxo observado é comumente de grau leve, e este não parece se associar à pior evolução clínica. No estudo de Lemos et al. ${ }^{1}$, contudo, $7 \%$ dos pacientes submetidos ao implante de CoreValve ${ }^{T M}$ apresentaram insuficiência aórtica moderada ou grave. De significado distinto, a ocorrência de insuficiência aórtica significativa $(>2+/ 4+)$ tem sido relacionada a pior prognóstico. Em um registro alemão $(n=690)$ com as próteses CoreValve $^{\mathrm{TM}}$ e Edwards-SAPIEN ${ }^{\mathrm{TM}}$, 17\% dos pacientes apresentaram insuficiência aórtica significativa pósimplante. ${ }^{11}$ Esses indivíduos evoluíram mais frequentemente com baixo débito cardíaco (20\% vs. 4,4\%), insuficiência respiratória (16,5\% vs. 7,1\%) e óbito $(15,1 \%$ vs. 6,7\%) em 30 dias; nessa casuística, a regurgitação significativa foi preditora de mortalidade aos 30 dias. Em grande registro italiano recentemente publicado, essa complicação também foi preditora de mortalidade entre 30 dias e 12 meses pós-implante [hazard ratio (HR) 3,57]. ${ }^{9}$ Os fatores implicados na ocorrência de regurgitação significativa parecem relacionar-se às características anatômicas próprias do complexo ânulo-aórtico (configuração circular ou elíptica, calcificação assimétrica), à desproporção entre o anel valvar e a prótese escolhida, e ao posicionamento incorreto da prótese ("muito baixa" ou "muito alta" em relação ao anel).

No relato de casos de Bernardi et al. ${ }^{2}$, uma das pacientes sofreu dois episódios de acidente vascular cerebral (sendo um nos primeiros 30 dias e outro tardio). Embora os estudos que utilizam ressonância magnética com difusão apontem para o surgimento de novas lesões cerebrais em mais da metade dos pacientes submetidos a implante percutâneo ${ }^{12}$, a incidência clínica de AVC varia de $1 \%$ a $10 \%$. No estudo PARTNER B, 6,7\% dos pacientes sofreram AVC ou ataque isquêmico transitório nos primeiros 30 dias e 10,6\% apresentaram a complicação aos 12 meses, taxas que foram significativamente mais altas que as observadas no grupo controle $(1,7 \%$ e $4,5 \%$, respectivamente). ${ }^{10}$ Mais facilmente compreendido, a etiologia do AVC que ocorre durante o procedimento se relaciona ao ateroembolismo de placas presentes na aorta ascendente e no arco aórtico. Postula-se que a contínua redução do perfil dos instrumentais e a utilização de novos dispositivos para proteção cerebral (EMBRELLA ${ }^{\mathrm{TM}}$ Edwards Lifesciences, e CLARET ${ }^{\mathrm{TM}}$, Claret Medical Inc., Santa Rosa, Estados Unidos) poderão ter impacto favorável nesse cenário. Outras potenciais causas podem envolver, contudo, a embolização de cálcio e debris dos folhetos da valva aórtica nativa, o tromboembolismo proveniente da prótese valvular e a fibrilação atrial. Assim, necessitamos de melhor compreensão a respeito dos mecanismos de AVC mais tardio e, principalmente, de respostas sobre qual a melhor forma de preveni-lo, com estudos que investiguem, por exemplo, riscos e benefícios da prescrição atualmente recomendada de terapia antiplaquetária dupla (aspirina e clopidogrel) por seis meses.

O implante percutâneo da bioprótese não exclui a valva nativa e leva à compressão dos folhetos, do anel valvar e de estruturas adjacentes. O acometimento do sistema de condução durante seu trajeto pelo septo interventricular pode se associar a bloqueio do ramo esquerdo e bloqueio atrioventricular total. Assim, o tratamento percutâneo pode requerer o implante de marca-passo definitivo em 3\% a 39\% dos pacientes. ${ }^{13-15}$ Uma vez que critérios definidores para a instalação de marca-passo nesse cenário ainda não estejam disponíveis, as taxas de utilização são, de certo, influenciadas pelo limiar de indicação em diferentes centros. Contudo, segundo estudos observacionais, as taxas de implante de marca-passo definitivo são maiores com a prótese CoreValve ${ }^{\mathrm{TM}}(9 \%$ a $36 \%)$, quando comparada à prótese Edwards-SAPIEN ${ }^{\mathrm{TM}}$ (3\% a $\left.12 \%\right)$. A maior protrusão da CoreValve ${ }^{T M}$ no trato de saída do ventrículo esquerdo e sua natureza autoexpansível, que pode determinar maior força radial sobre os tecidos subanulares, poderiam explicar tais achados. Na série de Lemos et al. ${ }^{1}, 22 \%$ dos pacientes foram submetidos a implante de marca-passo definitivo após CoreValve ${ }^{\mathrm{TM}}$, e esse fato não esteve relacionado a pior evolução clínica. A 
ausência de impacto clínico nessa casuística, contudo, pode ser explicada pelo número insuficiente de pacientes requeridos para que se demonstre a associação ou pela presteza com que esses indivíduos foram tratados ao desenvolver distúrbios de condução. A prática nos faz perceber que a ocorrência de bloqueios atrioventriculares predispõe a riscos; dessa forma, é necessária a identificação mais precisa de seus preditores (dentre os quais têm se destacado idade avançada, presença de bloqueio de ramo direito prévio e grau de calcificação valvar e de hipertrofia ventricular esquerda ${ }^{13-15}$ ) e de estratégias que reduzam sua incidência (pré-dilatação "não-agressiva", implante sem realização de pré-dilatação e melhor posicionamento em relação ao anel).

Por fim, a maioria dos estudos de seguimento clinico de pacientes que obtiveram implante adequado e sobrevida após 30 dias demonstra taxas de mortalidade consistentes com a faixa etária e as comorbidades da população que é atualmente tratada. $\mathrm{Na}$ experiência de Lemos et al. ${ }^{1}$, a taxa de sobrevida aos 12 meses foi de 79\%; nos registros publicados, sobrevida de $69 \%$ a $85 \%$ tem sido reportada. Cerca da metade dos óbitos no seguimento tardio são de etiologia nãocardíaca, o que ratifica a necessidade de selecionar pacientes que não só suplantem os riscos do procedimento, mas que possam também usufruir de seus benefícios a longo prazo, e esse talvez seja um de nossos maiores desafios. Embora a aplicação de escores, o acúmulo de conhecimentos a respeito dos parâmetros anatômicos, a experiência técnica adquirida e a reprodutibilidade dos resultados desse novo (e já maduro) procedimento contribuam sobremaneira para alcançarmos o estágio atual, selecionar adequadamente o paciente para o implante percutâneo de prótese valvular aórtica é a chave fundamental para seu sucesso. E, nesse aspecto, a ferramenta de maior importância continua sendo o julgamento clínico criterioso, com ênfase numa abordagem multidisciplinar, com o envolvimento de cardiologistas intervencionistas, clínicos, anestesistas e especialistas em imagens cardiovasculares, e a inclusão do cirurgião cardíaco desde a etapa inicial. Enquanto se espera por mais evidências, isso representa boa prática clínica.

\section{CONFLITO DE INTERESSES}

Os autores declaram não haver conflito de interesses relacionado a este manuscrito.

\section{REFERÊNCIAS}

1. Lemos PA, Saia F, Marzocchi A, Mariani Jr. J, Esteves Filho A, Kajita LJ, et al. Implante transcateter de prótese valvular aórtica: perfil clínico e evolução de uma série consecutiva de 75 casos do registro conjunto Universidade de Bolonha/ Hospital Sírio-Libanês. Rev Bras Cardiol Invasiva. 2011;19(1): 24-7.
2. Bernardi GLM, Sarmento-Leite R, Prates PRL, Quadros AS, Giusti I, Grando T, et al. Implante valvular aórtico percutâneo: dois anos de evolução da experiência inicial no Sul do Brasil. Rev Bras Cardiol Invasiva. 2011;19(1):28-33.

3. Leon M, Piazza N, Nikolsky E, Blackstone H, Cutlip DE, Kappetein AP, et al. Standardized endpoint definitions for transcatheter aortic valve implantation clinical trials: a consensus report from the Valve Academic Research Consortium. J Am Coll Cardiol. 2011;57(3):253-69.

4. Clavel MA, Webb JG, Pibarot P, Altwegg L, Dumont E, Thompson $\mathrm{C}$, et al. Comparison of the hemodynamic performance of percutaneous and surgical bioprostheses for the treatment of severe aortic stenosis. J Am Coll Cardiol. 2009; 53(20):1883-91.

5. Yan TD, Cao C, Martens-Nielsen J, Padang R, Ng M, Vallely $M P$, et al. Transcatheter aortic valve implantation for highrisk patients with severe aortic stenosis: a systematic review. J Thorac Cardiovasc Surg. 2010;139(6):1519-28.

6. Coeytaux RR, Williams JW Jr, Gray RN, Wang A. Percutaneous heart valve replacement for aortic stenosis: state of the evidence. Ann Intern Med. 2010;153(5):314-24.

7. Eltchaninoff $H$, Prat A, Gilard M, Leguerrier A, Blanchard D, Fournial G, et al.; FRANCE Registry Investigators. Transcatheter aortic valve implantation: early results of the FRANCE (FRench Aortic National CoreValve and Edwards) registry. Eur Heart J. $2011 ; 32(2): 191-7$.

8. Zahn R, Gerckens U, Grube E, Linke A, Sievert H, Eggebrecht $\mathrm{H}$, et al.; German Transcatheter Aortic Valve Interventions Registry Investigators. Transcatheter aortic valve implantation: first results from a multi-centre real-world registry. Eur Heart J. $2011 ; 32(2): 198-204$.

9. Tamburino C, Capodanno D, Ramondo A, Petronio AS, Ettori F, Santoro G, et al. Incidence and predictors of early and late mortality after transcatheter aortic valve implantation in 663 patients with severe aortic stenosis. Circulation. 2011; 123(3):299-308.

10. Leon MB, Smith CR, Mack M, Miller C, Moses JW, Svensson LG, et al.; PARTNER Trial Investigators. Transcatheter aorticvalve implantation for aortic stenosis in patients who cannot undergo surgery. N Engl J Med. 2010;363(17):1597-607.

11. Abdel-Wahab M, Zahn R, Horack M, Gerckens U, Schuler G, Sievert $\mathrm{H}$, et al.; German Transcatheter Aortic Valve Interventions Registry Investigators. Aortic regurgitation after transcatheter aortic valve implantation: incidence and early outcome. Results from the German Transcatheter Aortic Valve Interventions Registry. Heart. 2011 Mar 12. [Epub ahead of print]

12. Ghanem A, Müller A, Nähle CP, Kocurek J, Werner N, Hammerstingl $C$, et al. Risk and fate of cerebral embolism after transfemoral aortic valve implantation: A prospective pilot study with diffusion-weighted magnetic resonance imaging J Am Coll Cardiol. 2010;55(14);1427-32.

13. Jilaihawi $H$, Chin D, Vasa-Nicotera $M$, Jeilan $M$, Spyt $T, N g$ $\mathrm{GA}$, et al. Predictors for permanent pacemaker requirement after transcatheter aortic valve implantation with the CoreValve bioprosthesis. Am Heart J. 2009;157(5):860-6.

14. Piazza N, Onuma Y, Jesserun E, Kint PP, Maugenest AM, Anderson $\mathrm{RH}$, et al. Early and persistent intraventricular conduction abnormalities and requirements for pacemaking after percutaneous replacement of the aortic valve. JACC Cardiovasc Interv. 2008;1(3):310-6.

15. Khawaja MZ, Rajani R, Cook A, Khavandi A, Moynagh A, Chowdhary $S$, et al. Permanent pacemaker insertion after Corevalve transcatheter aortic valve implantation: - incidence and contributing factors (the UK CoreValve Collaborative). Circulation. 2011;123(9):951-60. 